\title{
Pratiques
}

Linguistique, littérature, didactique

175-176 | 2017

Didactiques et médiations des arts et de la littérature

\section{Survivre au prédicat : le cas du Québec}

Surviving the predicate: the case of Quebec

\section{Carole Fisher et Marie Nadeau}

\section{(2) OpenEdition}

Journals

Édition électronique

URL : http://journals.openedition.org/pratiques/3775

DOI : 10.4000/pratiques. 3775

ISSN : 2425-2042

\section{Éditeur}

Centre de recherche sur les médiations (CREM)

\section{Référence électronique}

Carole Fisher et Marie Nadeau, «Survivre au prédicat : le cas du Québec », Pratiques [En ligne], 175-176 | 2017, mis en ligne le 22 décembre 2017, consulté le 02 mai 2019. URL : http:// journals.openedition.org/pratiques/3775 ; DOI : 10.4000/pratiques.3775

Ce document a été généré automatiquement le 2 mai 2019.

(c) Tous droits réservés 


\title{
Survivre au prédicat : le cas du Québec
}

\author{
Surviving the predicate: the case of Quebec
}

Carole Fisher et Marie Nadeau

1 Il y a plus de vingt ans, É. Genevay (1996: 83) écrivait: «L'école d'aujourd'hui ne peut échapper à la nécessité de repenser la grammaire ». Si l'on se fie aux vives réactions qu'a récemment soulevées en France une tentative en ce sens, il semble bien que le chemin pour y arriver n'est pas de tout repos. Pourtant l'adoption d'un cadre grammatical moderne pour l'enseignement $d u$ français s'est bel et bien fait ailleurs dans la francophonie, notamment au Québec ${ }^{1}$. Nous nous proposons d'en témoigner ici, mais il faudra aussi constater que dans certaines zones, les changements se font à petits pas. C'est le cas pour la description de la phrase sur laquelle nous allons nous centrer ici, et dont nous suivrons l'évolution dans les programmes, puis dans un échantillon de matériel didactique. En dernière partie, à la lumière d'une recherche en cours, nous tenterons de montrer l'utilité de la notion de prédicat lorsqu'elle est mise au service d'apprentissages comme la ponctuation. Mais il convient d'abord de clarifier certains points essentiels à une bonne compréhension des choses.

\section{Langue, sciences du langage et grammaire}

2 Contrairement à une idée reçue, qui relève des représentations communes, la grammaire n'est pas la langue. En tant que locuteurs, ce que nous percevons de la langue, ou du langage, ce sont des réalisations : des énoncés, des textes, des discours, autrement dit les produits de l'emploi d'une langue particulière dans différentes circonstances et pour diverses fins. Par contre, ce qui rend possible ces productions, le " comment ça marche », échappe totalement au locuteur ${ }^{2}$. De nos jours, ce sont des disciplines qu'on regroupe sous le nom de « sciences du langage » qui élaborent des modèles explicatifs et descriptifs du fonctionnement du langage et des systèmes linguistiques. Dans un passé plus lointain, ce rôle revenait aux grammairiens. On aura compris qu'à l'instar d'autres domaines scientifiques, diverses théories explicatives peuvent coexister à propos d'un même objet 
langagier et, bien entendu, que ces théories évoluent avec le temps (voir par ex. Combettes, 2011 ou ici même, Elalouf).

3 Il en va de même de la grammaire. Comme discipline scolaire, on peut la définir comme un ensemble de connaissances reconnues nécessaires, à une époque donnée, pour comprendre minimalement le fonctionnement d'une langue, de manière à pouvoir l'écrire et la parler correctement. La grammaire ne se confond pas avec les théories linguistiques, mais il est normal et souhaitable qu'elle puisse en tirer parti pour le bénéfice de l'apprentissage. Par conséquent, la description que propose aujourd'hui une grammaire scolaire du français ne saurait être celle qui était proposée il y a un siècle ou deux, dans la mesure où les connaissances que l'on possède maintenant apparaissent plus précises et plus cohérentes et permettent de mettre davantage l'accent sur les régularités $\mathrm{du}$ fonctionnement de la langue.

\section{Grammaire traditionnelle - grammaire nouvelle}

4 Ce qu'on appelle la grammaire traditionnelle s'inscrit en fait dans le prolongement d'un courant grammatical, qui s'est développé, selon A. Chervel (1977), entre le milieu du XIX ${ }^{\mathrm{e}}$ siècle et 1920. Appelée par les nouveaux besoins de l'école, cette grammaire rompait avec la précédente en proposant une analyse plus complète de la phrase et en s'affranchissant du procédé du sous-entendu ${ }^{3}$ qui consistait, par exemple, à analyser Il est à Paris comme Il est demeurant à Paris. Mais comme l'indique A. Chervel (ibid., p. 171), c'est le fait de reconnaitre un statut grammatical au complément circonstanciel qui ouvre véritablement la voie à cette grammaire. À partir de là, en effet, il devient possible de dégager, de proche en proche, divers types de compléments et de fonctions, en s'appuyant notamment sur un système de questions : la question quoi pour identifier un complément direct, les questions où, quand, comment, pourquoi pour identifier un complément circonstanciel, etc. Se trouvent ainsi regroupées, sur une base sémantique, des constructions syntaxiques différentes, comme Max a travaillé hier/Max a travaillé la semaine dernière/Max a travaillé dès l'aube.

5 Assez rapidement, la différenciation des compléments circonstanciels va s'allonger, de sorte que M. Grévisse (1992, p. 38-39) en propose près de vingt dans le Précis, l'abrégé pour le milieu scolaire du Bon usage, cet ouvrage emblématique de la grammaire traditionnelle dont la première édition ${ }^{4}$ date de 1936. On y trouve par exemple des compléments circonstanciels de cause, d'instrument, de distance, de poids, de matière, de propos.

6 Une caractéristique importante d'une grammaire traditionnelle est ainsi de fonder la définition des parties du discours avant tout sur des critères sémantiques comme dans les deux exemples suivants tirés du Précis de grammaire française de M. Grévisse (1992) :

Le nom ou substantif est un mot qui sert à désigner les êtres, les choses, les idées.

(p. 57)

Le verbe est un mot qui exprime, soit l'action faite ou subie par le sujet, soit

l'existence ou l'état du sujet, soit l'union de l'attribut au sujet. (p. 134)

7 Identifier la «nature » des mots à partir de telles définitions conduit rapidement à des confusions. C'est sans doute l'exposition aux exemples qui aidaient les apprenants à ne pas prendre ces définitions au pied de la lettre et à reconnaitre un nom plutôt qu'un verbe dans orage, explosion, malade, ou une « qualité » dans second ou judiciaire. 
8 Par ailleurs, la grammaire traditionnelle se caractérise par deux niveaux d'analyse: l'analyse grammaticale, qui consiste à énumérer pour chaque mot d'une phrase sa nature et sa fonction, principalement pour assurer la réalisation des accords, et l'analyse logique dévolue à l'étude des rapports entre les " propositions » (subordonnées et principales). Le plan d'un manuel de grammaire traditionnelle se présente ainsi comme une suite de chapitres sur les différentes classes de mots (Nom, Verbe, Article, Adjectif, etc.) et une partie consacrée à la phrase complexe. En revanche, très peu d'attention est accordée à la structure de la phrase simple.

9 Avec le développement de la linguistique au milieu du siècle dernier, les faiblesses et les limites de la grammaire traditionnelle sont de plus en plus mises en lumière, tandis que de nouvelles descriptions de la langue «pour l'école » voient le jour (ex. : Genouvrier \& Peytard, 1970; Dubois \& Lagane, 1973; Genouvrier \& Gruwez, 1987). Ce nouveau cadre grammatical, découle, pour ce qui est de la grammaire de la phrase, d'une transposition didactique de la linguistique structurale et de la grammaire générative des années 1960-1970.

10 L'introduction de cette grammaire de la phrase en France, dans les années 1970, constitue pour C. Vargas (2011, p. 115) une véritable rupture pour la grammaire scolaire, qui vient modifier «les unités traitées (les groupes et non les mots)» de même que «les outils (formels et non plus sémantiques ou logiques) ». L'ouverture au texte et au discours comme objets d'analyses grammaticales sera plus tardive, elle date des années 1990, et constitue pour C. Vargas (2011) la «seconde révolution» dans la rénovation de la grammaire scolaire (voir, par ex. Genevay, 1994).

11 Trois principales notions caractérisent ce virage apporté par la grammaire dite « rénovée» ou «nouvelle». La notion de groupe, d'abord, qui reconnait un niveau d'organisation entre la phrase et ses unités, les mots. L'opération de substitution permet de délimiter ces groupes fonctionnels à l'intérieur d'une phrase comme dans l'exemple suivant :

Le chat de la voisine s'est sauvé sur le toit.

[Le chat de la voisine] [s'est sauvé sur le toit]

[il] [s'est endormi]

Une seconde notion fondamentale est celle de phrase de base, ou phrase minimale, qui est définie comme comportant obligatoirement deux groupes ordonnés : un Groupe nominal ${ }^{5}$ occupant la fonction de sujet suivi d'un Groupe verbal et, de manière facultative, un ou des groupes compléments de phrase, indépendants des deux premiers. Chacun de ces groupes est composé d'un noyau (ex. un nom dans le cas d'un GN) et d'éventuelles expansions (ex. pour le nom: déterminant, adjectif, groupe prépositionnel). La phrase apparait ainsi comme une structure de relations hiérarchisées, qui se prête à une représentation arborescente. La phrase de base constitue un modèle abstrait pour construire des phrases transformées (ex. : des phrases interrogatives, passives, avec subordonnées) et décrire ces transformations en comparant les phrases réalisées à ce modèle.

La troisième notion importante à mentionner est celle de manipulation syntaxique. Au jeu des questions de la grammaire traditionnelle, qui était loin de mener les élèves à des analyses justes (ex. Il semble malade: il semble quoi ? - malade, COD*), la grammaire rénovée oppose des opérations qui découlent de la description des classes de mots sur la base de leurs propriétés morphologiques et syntaxiques (et non plus sémantiques). Ainsi, puisque l'adjectif est une expansion du nom et qu'il peut recevoir un adverbe quand il est 
qualifiant, on peut recourir aux manipulations d'effacement et d'addition afin de vérifier si un mot (ici jeunes) est un adjectif :

Les jeunes conducteurs sont téméraires.

$\Rightarrow$ Les $\emptyset$ conducteurs sont téméraires. (la phrase demeure acceptable)

$\Rightarrow$ Les très jeunes conducteurs sont téméraires. (même chose)

Par contre, l'hypothèse que «conducteurs » est un adjectif ne serait pas vérifiée en testant l'addition d'un adverbe :

$\Rightarrow{ }^{*}$ Les jeunes très conducteurs sont téméraires. (la phrase ne se dit pas)

L'encadrement par C'est/Ce sont... qui est une autre manipulation, facile à utiliser et souvent plus sure pour repérer le sujet d'une phrase que la question qui/qu'est-ce qui ? Exemple :

Les responsables de l'entreprise réunissent des fonds.

Question: «qui est-ce qui réunissent?» se dit mal et risque de se confondre avec

"qu'est-ce qu'ils réunissent?»

Manipulation : ce sont [les responsables de l'entreprise] qui réunissent...

Certes, la grammaire rénovée n'est pas parfaite, certaines constructions ou faits de langue demeurent difficiles à analyser ou à bien différencier ${ }^{6}$. De plus, toute grammaire scolaire étant une transposition didactique, certains choix paraitront toujours discutables pour le spécialiste de la linguistique, et selon le courant théorique adopté. Néanmoins, ce cadre grammatical apparait dans son ensemble plus cohérent et mieux fondé que la grammaire traditionnelle. En plaçant la phrase au cœur de la description, cette grammaire permet aux élèves de comprendre les grandes régularités des structures du français (phrase de base, types et formes de phrases, phrases à construction particulière), de comprendre les relations entre les mots d'une phrase (relations donneurs/receveurs) et ainsi d'acquérir des repères plus surs pour écrire et comprendre des textes.

\section{La grammaire nouvelle dans la succession des programmes québécois}

Ce n'est qu'en 1995 que la grammaire rénovée fait officiellement son entrée au Québec, par la voie du programme de français du secondaire (MEQ, 1995). Il s'agit d'un changement de cap très important, à la fois par le degré d'explicitation de ce programme, comparé au précédent (centré sur la communication et qui donnait lieu à une pratique " occasionnelle » de la grammaire, et par la rigueur que les auteurs ont voulu y mettre ${ }^{7}$ au plan notionnel et terminologique. À côté des parties sur l'écriture, la lecture et la communication orale, une partie du programme est consacrée au fonctionnement de la langue, ce qui comprend le lexique, la grammaire de la phrase et du texte, l'orthographe grammaticale et la conjugaison, l'orthographe d'usage et la terminologie à employer avec les élèves. Avec ce programme, l'enseignement de la grammaire doit être "systématique ", tandis que les connaissances sur la langue et les textes doivent trouver « leur application dans des textes lus, racontés, dits ou écrits par l'élève » (MEQ, 1997b, p. 5).

Pour ce qui est de la grammaire de la phrase et du texte, l'organisation des contenus se fait en partant des différents groupes constitutifs de la phrase pour arriver au texte, en passant par les subordonnées, les types et formes de phrases, ainsi que les liens dans et entre les phrases (juxtaposition, coordination) et la ponctuation. Les choix grammaticaux sont clairs, avec les notions de classes et groupes de mots, de complément de phrase, de déterminants, de manipulations linguistiques. L'appareil terminologique est par ailleurs 
allégé : la notion de complément est élargie à tout mot sous la dépendance d'un autre, les compléments du verbe sont nommés $\mathrm{CD}$ et $\mathrm{CI}$, on ne parle plus de compléments circonstanciels, ni d'adjectifs possessifs ou démonstratifs qui font partie des déterminants.

On remarque, par contre et assez curieusement, que c'est uniquement dans la courte présentation de cette section sur la grammaire de la phrase et du texte qu'il est fait mention de la phrase de base :

une phrase déclarative, positive, active et neutre dans laquelle les groupes sont placés dans l'ordre suivant: groupe du nom sujet + groupe du verbe + groupes facultatifs. (MEQ, 1997a, p. 1318)

Tandis que son utilisation comme base de comparaison pour s'assurer qu'une phrase réelle est bien construite se limite aussi à une simple mention dans la présentation et dans une note :

Pour analyser chaque phrase [...], on la compare à la phrase de base : on repère et vérifie la présence des groupes obligatoires, on observe l'ordre de ces groupes et la ponctuation qui accompagne les déplacements des groupes ainsi que les marques propres au type, à la forme ou à la construction particulière. (ibid., p. 150)

Il en va de même de la notion de phrase syntaxique qui figure dans la présentation (opposée à la phrase graphique, décrite sans être nommée), mais le mot n'apparait pas dans la liste des termes à utiliser avec les élèves.

Il n'en demeure pas moins que ce programme de 1995 pour le secondaire proposait un contenu grammatical consistant, en totale rupture avec celui de la grammaire traditionnelle. En outre, il suggérait un mode de travail avec les élèves qui s'appuyait sur l'observation de régularités de la langue, l'identification de phénomènes langagiers et l'évaluation de leur apport à la phrase ou au texte. Il se distinguait aussi fortement du programme, adopté l'année précédente pour le primaire (MEQ, 1994), qui proposait bien une analyse de la phrase en termes de groupes et de constituants, reconnaissait la classe des déterminants, mais reconduisait des notions de la grammaire traditionnelle comme la proposition et le complément circonstanciel. Savoir analyser une phrase semblait aussi de peu d'utilité pour aider l'élève à délimiter les frontières d'une phrase et la ponctuer, le programme suggérant plutôt de "s'appuyer sur le sens et sur sa connaissance de la langue parlée pour repérer les phrases » (ibid., p. 32).

23 Les quelques éléments de grammaire rénovée qui figuraient dans ce programme de 1994 pour le primaire vont donc passer inaperçus. Le programme suivant, pour cet ordre d'enseignement, apparait en 2001 dans la foulée d'une vaste réforme pédagogique qui adopte une approche par compétences: le Programme de formation de l'école québécoise (PFEQ) (MELS, 2001). Dans la section sur le français langue d'enseignement, la nouvelle grammaire ressort plus clairement que dans le programme précédent (par exemple : la notion de complément de phrase est à travailler au $3^{\mathrm{e}}$ cycle). Toutefois, la notion de phrase de base demeure absente et aucune manipulation syntaxique n'est mentionnée. Mais le plus notable dans ce programme, est que les contenus en grammaire et en orthographe pour toutes les années du primaire se trouvent dans une liste de « savoirs essentiels » qui tient en trois pages (ibid., p. 88-90)! Pas étonnant alors que les éditeurs de matériel scolaire aient donné peu d'écho à l'introduction de notions de la grammaire rénovée dans les ouvrages parus à cette époque, d'autant plus que devant l'ampleur des changements introduits par la réforme, les formations offertes aux enseignants ont porté sur des 
aspects plus larges du renouveau pédagogique, en laissant dans l'ombre l'enseignement de la grammaire.

La controverse entourant le "renouveau pédagogique » et l'insatisfaction devant les difficultés récurrentes des élèves en écriture conduisent la Ministre de l'époque à déposer au début de l'année 2008 un Plan d'action pour l'amélioration du français. Une des mesures importantes de ce plan consiste à faire préciser les contenus d'apprentissage en écriture pour chacune des années du primaire, afin de pallier les lacunes d'un programme beaucoup trop vague et organisé, de surcroit, par cycle de deux ans. Un comité formé de conseillers pédagogiques et de représentants de l'association québécoise des professeurs de français (AQPF) et de l'association internationale pour la recherche en didactique du français (AIRDF) se met au travail et, l'année suivante, la Progression des apprentissages en écriture parait (MELS, 2009) ${ }^{9}$. Des trois pages de «savoirs essentiels » du programme de 2001 , on passe à 60 pages de précisions avec ce document qui complète officiellement le $\mathrm{PFEQ}^{10}$. La nouvelle grammaire devient alors incontournable au primaire et, pour plusieurs enseignants de ce niveau scolaire, il s'agit de leur premier véritable contact avec cette grammaire.

Dans la section sur les accords, on remarque la distinction entre classes de mots variables et invariables, et l'introduction des notions de donneur/receveur. Les manipulations syntaxiques sont non seulement évoquées, mais il est nécessaire de les distinguer et de connaitre l'utilité de chacune. Par exemple, dans la section des connaissances sur la syntaxe et la ponctuation, il s'agit de: «connaitre des manipulations pour identifier le groupe de mots qui occupe la fonction SUJET » et on énumère : l'encadrement par C'est... qui, le remplacement par un pronom de conjugaison, le non-effacement, le nondéplacement (ibid., p. 43). La phrase est au cœur de cette même section. Parmi les caractéristiques d'une phrase contenant un seul verbe conjugué, on note «la présence d'un SUJET, d'un PRÉDICAT et parfois d'un ou de COMPLÉMENTS DE PHRASE » (ibid., p. 41). Connaitre les caractéristiques de la phrase de base figure aussi au tableau :

i. une phrase de type déclaratif et de forme positive

ii. une phrase dont les constituants sont placés dans l'ordre suivant : un SUJET + un

PRÉDICAT + (un ou des COMPLÉMENTS DE PHRASE) (p. 44)

Tout comme en connaitre l'utilité: «un outil de comparaison pour observer et comprendre la structure de diverses phrases » (p. 45).

Du côté du secondaire, le nouveau programme adopté dans le cadre du PFEQ, entre 2005 et 2007, ne propose rien au chapitre de la grammaire, mais reconduit « les orientations de celui de 1995 et privilégie la description de la langue que préconisent les grammaires dites nouvelles » (MELS, 2005, p. 92). Dans la liste des «éléments d'apprentissage », on voit néanmoins la notion de phrase de base, mais simplement définie dans une note.

C'est donc la Progression des apprentissages au primaire qui marque un jalon décisif dans l'adoption et l'explicitation d'un cadre de grammaire moderne pour enseigner le français au Québec. À partir de là, la définition de la phrase est formulée en termes de Sujet et de Prédicat, et non plus de GN Sujet et de GV. À la fonction de Sujet répond celle de Prédicat, avec leurs possibles réalisations respectives :

SUJET + PRÉDICAT

(GN) (Pro) (GInf.) (Sub. compl.) GV

Le plan des fonctions est alors clairement distingué de celui des groupes syntaxiques qui peuvent les remplir. Car ainsi que l'explique E. Bulea Bronckart (2015, p. 40) : 
la grammaire rénovée tente de clarifier au maximum la différence de niveaux entre :

- les classes grammaticales (nom, verbe, préposition, etc.) ;

- les syntagmes ou groupes qui organisent les rapports d'interdépendance entre ces classes (groupe nominal, groupe verbal, groupe prépositionnel, etc.) ;

- les fonctions syntaxiques que les classes et les groupes remplissent (sujet, prédicat, complément de phrase, complément du nom, etc.) ;

- les valeurs sémantiques de ceux-ci (temps, lieu, cause, conséquence, etc.);

- enfin, [...] au-delà du modèle PHRASE P, les fonctions textuelles /discursives

Il semble que ce principe ait mis du temps à s'imposer dans l'adoption de la grammaire rénovée dans les programmes québécois. Mais qu'en est-il du matériel didactique et des grammaires : la notion de prédicat s'y rencontre-t-elle?

\section{La notion de prédicat dans le matériel didactique et les grammaires}

31 Comme on vient de le voir, la délimitation d'un cadre de grammaire moderne, avec ses notions et sa terminologie, ne s'est faite que graduellement dans les programmes québécois. Pour nous en tenir à la définition de la phrase, la notion de prédicat ne prend vraiment sa place qu'avec la Progression des apprentissages au primaire en 2009 et dans celle du secondaire en 2011. Cette notion est pourtant bien présente dans les grammaires parues avant la réforme des programmes de 2001 comme la Grammaire pédagogique du français d'aujourd'hui (Chartrand et al., 1999), ouvrage destiné au secondaire et largement utilisé en formation des enseignants, et Grammaire de base (Chartrand \& Simard, 2000), parue l'année suivante pour les élèves du primaire. On peut ainsi lire dans cette dernière : "Le premier constituant obligatoire de la phrase est le sujet" et "Le deuxième constituant obligatoire de la phrase est le prédicat »; on précise ensuite que la fonction de sujet est « le plus souvent remplie par un groupe du nom » ou par un pronom, tandis que celle de prédicat est « toujours remplie par un groupe du verbe» (ibid., p. 160-161).

On ne retrouve guère cette terminologie dans le matériel didactique produit dans le cadre du renouveau pédagogique qui suit (MELS, 2001). Il est instructif de comparer la manière de décrire la phrase et la terminologie retenue pour référer à ses deux groupes obligatoires dans une sélection de matériel (ensembles didactiques et grammaires) destiné au $3^{\mathrm{e}}$ cycle du primaire ( $5^{\mathrm{e}}$ et $6^{\mathrm{e}}$ années) ${ }^{11}$, avant et après la parution de la Progression des apprentissages au primaire (cf. Tableau 1).

Tableau 1. Métalangage relatif à la description de la phrase dans le matériel didactique et les grammaires avant et après la parution de la Progression des apprentissages au primaire (2009)

\begin{tabular}{|c|c|c|c|}
\hline \multicolumn{2}{|c|}{ CONTENU ET TERMES } & $\begin{array}{l}\text { Avant : éditions } \\
\text { de } 2002 \text { à } 2004 \\
\mathbf{N}=\mathbf{8}\end{array}$ & $\begin{array}{l}\text { Après : éditions } \\
\text { de } 2010 \text { à } 2015 \\
\mathbf{N}=\mathbf{8}\end{array}$ \\
\hline \multicolumn{2}{|c|}{ Définition de la $\mathrm{P}$ en 3 groupes } & 8 & 7 \\
\hline $\begin{array}{l}\text { Mention de } \\
\text { modèle }\end{array}$ & se de base comme & 4 & 3 \\
\hline Termes pour & GN-S & 2 & 1 \\
\hline
\end{tabular}




\begin{tabular}{|l|l|l|l|}
\cline { 2 - 4 } & GS & 6 & 1 \\
\cline { 2 - 4 } & Sujet & 2 & 7 \\
\hline Termes pour le $2^{\mathrm{e}}$ & GV & $\mathbf{8}$ & $\mathbf{8}$ \\
\hline Emploi du terme « Prédicat » & 3 & 7 \\
\hline Mention de la fonction du GV & 4 & 5 \\
\hline
\end{tabular}

La description de la phrase en termes de constituants, dont deux obligatoires, se retrouve dans l'ensemble du matériel. Toutefois, cette présentation n'est liée à la notion de phrase de base que dans deux ouvrages (\#4 et \#6) ${ }^{12}$ tandis qu'un autre parle d'un «modèle de base " à propos de la phrase déclarative (\#5) et qu'un dernier parle de "phrase modèle " (\#8). À l'exception d'une grammaire (\#6), qui oppose la phrase de base à la phrase transformée, la description de la phrase emprunte donc la terminologie de la grammaire nouvelle, mais sans distinguer le modèle abstrait qu'est la phase de base des phrases effectivement réalisées, comme le montre cette formulation : «La phrase est constituée de groupes de mots dont deux groupes de mots obligatoires [...]» (\#3). Il faut toutefois préciser que ni la notion de phrase de base ni celle de prédicat ne figuraient dans la liste des « savoirs essentiels » du PFEQ. Lorsque la description est plus développée, il arrive qu'on juxtapose des éléments de grammaire traditionnelle et de grammaire nouvelle, en évoquant que la phrase commence par une majuscule et se termine par un point, que c'est une suite de mots ordonnée qui a du sens, et qu'elle est formée de deux constituants obligatoires (\#7).

Pour ce qui est de la distinction entre le GV et sa fonction dans la phrase (ex. « ce qu'on

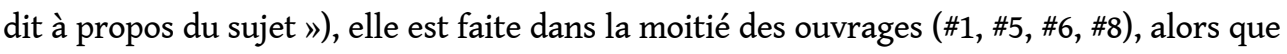
cette fonction n'est nommée par le mot prédicat que dans les trois derniers. Finalement, on remarque un certain flottement dans la manière de nommer le premier constituant de la phrase : le plus souvent groupe sujet (qui fait la paire avec le groupe $d u$ verbe), mais aussi groupe du nom sujet (\#2 et \#6), utilisé plus systématiquement dans le deuxième cas avec le symbole GN-S. Se trouve alors combinées la nature du groupe et sa fonction, ce qui n'est pas le cas avec une autre grammaire (\#8) qui utilise les termes "groupe sujet » et "groupe prédicat». Quant au terme sujet, il n'apparait que dans deux ouvrages (\#5, \#6) en vis-à-vis de prédicat.

À la suite de la publication de la Progression des apprentissages au primaire, le Ministère n'a pas appelé à la publication de nouveau matériel. Un seul ensemble (\#13) a reçu l'approbation ministérielle ${ }^{13}$, avec la réédition d'une grammaire que nous avons retenue ici (\#14). Les autres ouvrages correspondent souvent à du matériel périssable, des cahiers mêlant informations et activités pour les élèves. Sauf dans un ouvrage qui n'en souffle mot (\#12), on trouve une description de la phrase en constituants. Par contre, cette définition se rattache encore très peu à la notion de phrase de base qui figure pourtant bel et bien dans la Progression des apprentissages, au $3^{\mathrm{e}}$ cycle, parmi les connaissances liées à la " phrase de type déclaratif» (E.5, p. 44). Bien plus, dans un matériel (\#11) et dans la réédition de Grammaire de base (\#14), on observe des définitions qui amalgament phrase graphique et phrase syntaxique dans l'énumération des caractéristiques de la phrase : 
suite de mots ordonnés liés les uns aux autres [...] La P exprime quelque chose ; elle doit avoir un sens [...] Elle commence par une majuscule et se termine par un point [...] La P est obligatoirement formée d'un groupe sujet et d'un groupe du verbe...

(\#11, p. 71) progression. D'une part, l'entrée en scène du terme prédicat ( 7 fois sur 8 ), associé au rôle joué par le GV, et dont le sens est généralement précisé (\#12, \#14,\#15, \#17, \#18), comme ici : «Le prédicat indique généralement ce qu'on dit du sujet» (\#15). D’autre part, un changement dans le métalangage servant à désigner le premier constituant obligatoire de la phrase. Le terme hybride de Groupe sujet (ou GS) n'apparait plus que dans un seul matériel (\#11) alors qu'on trouve dans 7 ouvrages la désignation par la fonction seule (sujet ou Sujet) ou la combinaison nature du groupe et fonction (groupe du nom sujet, dans \#12). Ce qui n'empêche pas la paire Groupe du Nom/Groupe du verbe de servir encore à désigner les deux constituants obligatoires de la phrase, en parallèle avec une autre dénomination (\#11, \#12,\#13,\#14, \#15), ou parfois même de manière exclusive (\#17).

Ce survol de la terminologie utilisée dans le matériel didactique illustre à quel point, en dépit de quelques précurseurs, les changements en matière de grammaire sont lents et, souvent, mal assurés, car les contenus présentés (définition, terminologie, exemples) ne s'appuient pas toujours sur une réelle compréhension de la logique qui les fonde. Le souci des auteurs de demeurer simples auprès des élèves, ou la frilosité de leurs éditeurs face à l'innovation, peut aussi affecter la cohérence de ces contenus d'apprentissage.

Le pas suivant, que nous ne franchirons pas, consisterait à se demander ce qu'il en est de l'enseignement grammatical donné dans les classes. N'ignorant pas la distance entre prescriptions officielles et pratiques effectives, puis entre celles-ci et les apprentissages réalisés (cf. Bru, Altet \& Blanchard-Laville, 2004), il serait présomptueux de penser que la notion de prédicat est communément enseignée et utilisée dans les classes du Québec. Notre expérience nous apprend que le mot lui-même rebute encore certains enseignants, du moins jusqu'à ce qu'ils comprennent concrètement l'utilité de la notion. Pour qu'un programme s'actualise dans les classes, il est nécessaire que les enseignants s'en approprient le sens d'une manière pratique, autrement dit qu'ils comprennent et apprennent quoi en faire pour que les élèves en bénéficient. Ceci ne peut se réaliser sans un accompagnement adéquat, comme le démontrent plusieurs recherches sur le changement professionnel (cf. la revue de M.-H. Giguère, 2015) ou le rapport The New Teacher Project (TNTP, 2015), une étude américaine menée à grande échelle sur le cheminement professionnel des enseignants.

\section{L'exemple de la ponctuation pour illustrer l'utilité de la notion de prédicat}

En tant que constituant de la phrase de base, le prédicat peut servir, de concert avec les autres constituants, à appréhender la notion de phrase, une notion fortement polysémique ${ }^{14}$ qui pose problème aux élèves en écriture, tout particulièrement dans la ponctuation de leurs textes. Dans cette section, nous illustrerons d'abord ces difficultés à partir de textes d'élèves puis nous montrerons comment la notion de prédicat peut être utilisée avec eux pour mieux comprendre et expliquer la ponctuation des phrases qu'ils écrivent. 
$\mathrm{Au}$ Québec, des évaluations nationales en écriture ont lieu en $4^{\mathrm{e}}$ et $6^{\mathrm{e}}$ primaire ainsi qu'en $2^{e}$ et $5^{\mathrm{e}}$ secondaire. Il s'agit d'écrire un texte d'un genre adapté au niveau scolaire. Notons que la consultation d'ouvrages de référence est permise au cours de ces épreuves (dictionnaire, grammaire). Pourtant, dans ces examens d'écriture du MELS ${ }^{15}$, parmi les cinq critères évalués, le critère "syntaxe-ponctuation » est, à tous les niveaux scolaires, le $2^{e}$ critère pour lequel le taux d'échec est le plus élevé (MELS, 2012). Nous observons effectivement des difficultés dans la segmentation et la connexion des phrases chez des élèves de fin primaire et début secondaire en milieux défavorisés où nous sommes intervenues dans le cadre d'un projet de recherche ${ }^{16}$. Les extraits suivants de textes d'élèves, reproduits tels quels, en témoignent.

41 1) extrait du texte d'un élève de $5^{\mathrm{e}}$ année primaire (10-11 ans) qui raconte son voyage aux chutes Niagara :

[...] pour aller dans les chutes. Et je ne saurais pas d'écrire la sensation que j'ai eu quand ont étaient à l'intérieur des chutes. Mais ces comme si je resantai la récompense que l'eau nous a donné je l'ai resenti avec une tel puisance, il *a pas de mots c'était juste magique. Et recevoir l'eau sur mon visage cette juste incroyable. Et pour finir cette journée inoubliable ont a mangé dans un restaurant luxueux qui donnait à tous toronto. Et vers $8 \mathrm{~h} 30$ les lumières des chutes se sont allumé, et je n'ai qun mots à dire [...]

Du point de vue de la syntaxe et de la ponctuation, en laissant de côté l'orthographe, ce texte présente plusieurs phrases complexes bien construites, mais aussi de la surcoordination par et, tandis que plusieurs marques de ponctuation manquent: en particulier la virgule qui détache le complément de phrase lorsque déplacé devant le sujet.

2) extrait du texte d'un élève de $1^{\text {re }}$ secondaire (12-13 ans)

Il avait un pelage de gorille de couleur orange et en plus son poil était recouverte de sang, ce monstre avait 4 pattes de rhinocéros avec des griffes de phénix très aiguiser qui pouvais agripper la chair et en plus il avait tellement de sang sur ses griffe que mon sang était glacer.

44 La seule ponctuation de ce passage est une virgule. On remarque aussi la présence de surcoordinations (et en plus) pour marquer avec insistance, parfois maladroitement, ce qui pourrait être un début de phrase graphique (De plus, ...).

3) extrait du texte d'un autre élève de $1^{\text {re }}$ secondaire (12-13 ans)

... Après que le monstre nous ailes terroriser $\mathrm{j}$ 'ai aperçu un pistolet sur le plancher je l'ai pris j'ai tiré un coup de feu sur le monstre, mais il n'ait pas mort ses à ce moment que j'ai compris qu'il était invincible

Cet extrait d'un élève de 12-13 ans démontre une meilleure maitrise de l'écrit que le précédent, avec des phrases bien construites et sans surcoordination, mais la ponctuation y est encore défaillante : présence d'une seule virgule et aucune marque de fin de phrase.

47 Ces trois extraits montrent que les difficultés de segmentation/connexion de phrases observées en $5^{\mathrm{e}}$ année primaire (10-11 ans) persistent en $1^{\text {re }}$ secondaire (12-13 ans), du moins chez plusieurs élèves. Puisque la notion de phrase graphique (celle qui est ponctuée) ne semble pas encore acquise à ce stade de la scolarité, malgré un enseignement récurrent des règles élémentaires de ponctuation, il convient de trouver des dispositifs plus efficaces pour travailler cette notion en classe.

Prenant appui sur les fondements des dictées métacognitives (Cogis et al., 2016; Cogis, Fisher \& Nadeau, 2015 ; Fisher et Nadeau, 2014) et de ses effets positifs sur la maitrise de 
l'orthographe grammaticale (Wilkinson, 2009; Nadeau \& Fisher, 2014 ; Ammar \& Hassan, 2017), nous avons développé un dispositif pour travailler la ponctuation à la manière de la "phrase dictée du jour». L'objectif est de favoriser la verbalisation et la réflexion métalinguistique des élèves à propos de la ponctuation. Un court texte sans majuscule ni ponctuation est remis aux élèves qui doivent ajouter toutes les marques requises. L'enseignant inscrit ensuite au tableau l'ensemble des marques mises par les élèves (figure 1) :

Quelle ponctuation as-tu écrite à quel endroit?

Zac a mis une virgule ici, ceux qui en ont mis une aussi : levez la main.

Qui n'a mis aucun signe à cet endroit ? (l'absence de signe est signalée par $\varnothing$ )

Figure 1. Exemple de signes proposés par des élèves de $1^{\text {re }}$ secondaire et reportés au tableau lors d'une activité de ponctuation à la manière d'une phrase du jour

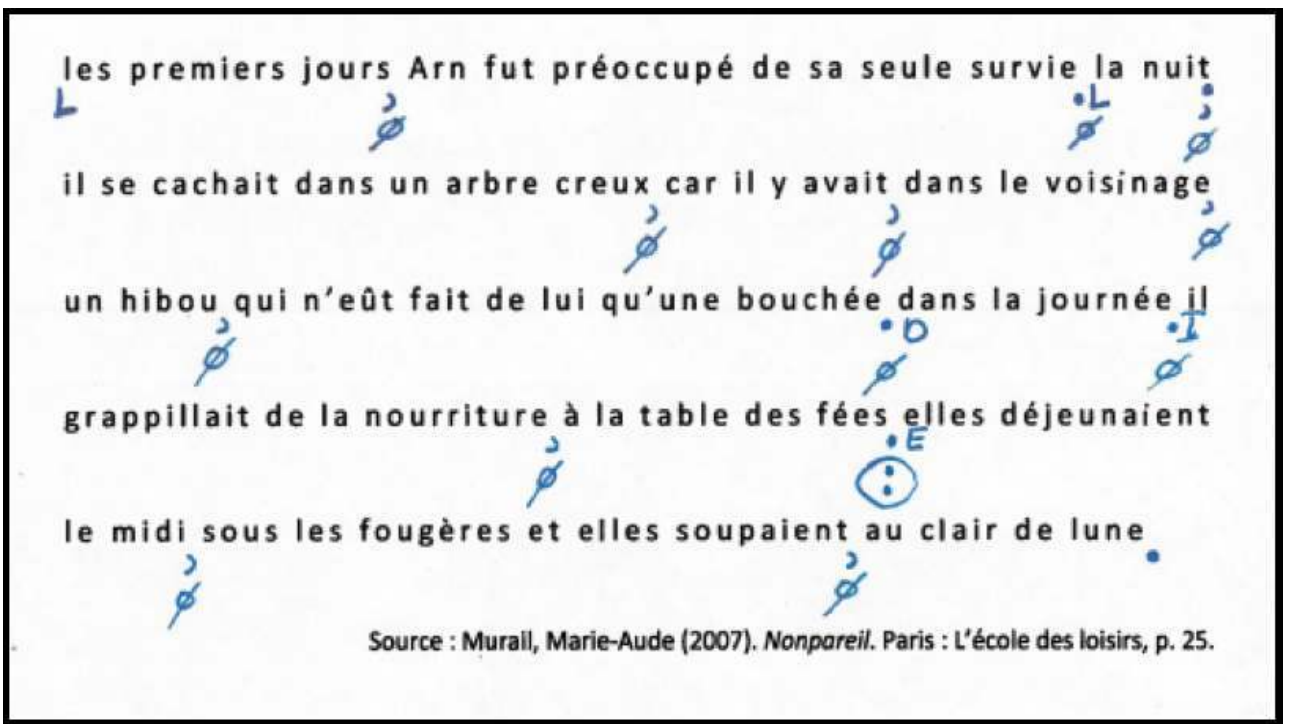

L'enseignant anime alors une discussion grammaticale sur chaque signe :

Pourquoi as-tu mis un point/une virgule à cet endroit?

Pourquoi penses-tu qu'aucun signe n'est nécessaire ici?

Comment sais-tu que la phrase se termine ici?

Quels sont les constituants de cette phrase?

Ce qui suit forme-t-il une autre phrase ? Pourquoi ?

53 C'est ainsi que les élèves sont amenés peu à peu à justifier la ponctuation qu'ils ont utilisée à partir de leurs connaissances grammaticales et non sur la base de leur intuition ou des fameuses pauses de l'oral. L'analyse des constituants entre alors en jeu et sert de preuve pour délimiter la fin d'une phrase puisqu'il faut minimalement un sujet et un prédicat ${ }^{17}$ pour en former une. Chaque constituant identifié doit être justifié à l'aide d'une manipulation syntaxique (encadrement du sujet par C'est ... qui; détachement du CdeP par l'ajout de et cela se passe; remplacement du prédicat par un verbe intransitif ou identification du verbe et de ses compléments essentiels). En ce qui concerne les signes de ponctuation, il est clair que divers signes sont souvent acceptables, donc acceptés (ex. : le point ou les deux points après fée dans l'exemple précédent).

Un complément de phrase ne peut être isolé des deux constituants obligatoires car il ne forme pas une phrase syntaxique autonome : par exemple, le GN la nuit dans l'exemple cidessus doit être rattaché à un ensemble S+Préd. Une discussion sur le sens du texte 
intervient ici pour déterminer s'il s'agit du CdeP de «Arn fut préoccupé de sa seule survie la nuit » (donc un point après nuit) ou du CdeP de la phrase suivante : «La nuit, il se cachait dans un arbre creux... ». C'est alors la compréhension en lecture qui intervient et qui s'en trouve approfondie.

Par l'analyse des constituants (sujet, prédicat, complément de phrase), les élèves observent rapidement diverses combinaisons qui permettent d'inclure plusieurs sujets et prédicats dans une seule phrase graphique. Il s'agit alors soit de juxtaposition de phrases syntaxiques autonomes (nécessitant une virgule), soit de coordination ou de subordination de phrases qui nécessitent une conjonction ou encore un pronom relatif (ex. : La nuit, il se cachait [...], car il y avait [...] un hibou qui [...]). Il est à noter que les conjonctions et les pronoms relatifs courants peuvent être repérés par les élèves comme simple moyen pour composer des phrases graphiques élaborées sans connaitre les types de subordonnées ni la distinction entre subordination et coordination. Une telle analyse est donc à la portée des élèves de $5^{\mathrm{e}}$ primaire.

Un autre cas pour lequel l'identification du prédicat intervient dans ce type d'activité concerne la virgule autour du complément de phrase. Un CdeP qui suit un prédicat ne requiert aucune ponctuation (ex. : Il grappillait de la nourriture dans la journée.) tandis qu'elle est obligatoire lorsque le CdeP se trouve déplacé par exemple devant le sujet (Dans la journée, il grappillait de la nourriture.).

L'analyse des constituants, dont le prédicat, occupe ainsi une place centrale dans ce dispositif qui maintient les élèves fort actifs sur le plan cognitif :

- ils observent diverses structures de phrases,

- ils verbalisent leurs raisonnements grammaticaux (ce qui leur permet entre autres d'exprimer de fausses conceptions et de les rectifier),

- ils rappellent les règles de ponctuation apprises (pour les avoir plus fraichement en mémoire au moment d'écrire),

- ils établissent des liens entre les «leçons de grammaire» et l'utilité des notions pour résoudre un problème d'écriture,

- ils discutent de manière approfondie du sens du texte et de certaines nuances qu'apportent diverses ponctuations acceptables dans une même phrase.

Le repérage des constituants de la phrase syntaxique (à l'aide des manipulations) est ainsi mis au service de la résolution de problèmes de ponctuation dans des textes de littérature jeunesse ou dans des extraits de textes d'élèves, ce qui rapproche ces derniers d'une situation de révision de texte. L'hypothèse selon laquelle ces exercices amènent les élèves à une meilleure maitrise de la ponctuation dans leurs textes sera testée sous peu dans le cadre de la recherche en cours.

\section{Conclusion}

Quelles leçons peut-on tirer de ce portrait de la notion de prédicat dans l'enseignement grammatical au Québec? Nous en voyons trois principales.

D'abord, les changements dans tout ce qui touche à la langue ne se font jamais sans heurts dans la mesure où elle fait partie de la culture, voire même de l'identité. Bien que la grammaire scolaire ne soit qu'une description vulgarisée de la langue, sa modernisation suscite toujours de la controverse chez les enseignants comme chez les parents qui ne se 
sentent plus aptes à aider leurs enfants dans cette matière, alors qu'ils sont plutôt admiratifs des connaissances en chimie ou en biologie de leurs jeunes du secondaire.

Par ailleurs, les changements, dans la sphère grammaire, se font lentement, à petits pas. Comme le montre l'examen de la terminologie dans le matériel didactique, les précurseurs ne sont pas immédiatement suivis, l'ancienne terminologie cohabite avec la nouvelle, même lorsque le métalangage rénové se trouve officiellement prescrit dans les programmes. La distance est souvent encore plus grande entre les prescriptions officielles et ce qui peut être réalisé dans en classe. Le fait est que le virage vers la grammaire nouvelle n'est pas qu'un simple changement d'étiquettes. Pour l'enseignant, cette nouvelle grammaire amène une autre manière de s'en servir et il doit être formé en conséquence, ce que les premières formations au Québec n'ont pas du tout fait. De plus, puisque apprendre, comme le pense P. Meirieu, c'est acquérir un savoir autant que donner du sens à ce savoir, l'enseignant doit également en percevoir les bénéfices pour l'élève.

62 Enfin, nous sommes convaincues que tout enseignant désire profondément voir ses élèves progresser. Si les pratiques touchant l'enseignement de la grammaire ne changent pas suffisamment, ou pas assez rapidement, il faut y voir non pas de la mauvaise volonté ou de la paresse mais une défaillance de la formation initiale et continue. Nos propres recherches depuis une dizaine d'années, sur les dictées métacognitives puis sur la syntaxe et la ponctuation à la manière de la phrase dictée du jour, font la preuve qu'en fournissant un accompagnement adéquat aux enseignants, ces derniers réussissent à opérer un changement durable de leurs pratiques en grammaire qui profite aux élèves. Cet accompagnement adéquat n'est pas une formule magique! Il s'appuie sur les constats des recherches récentes sur le cheminement professionnel. Il serait donc possible pour les décideurs de mettre en place ces conditions gagnantes.

\section{BIBLIOGRAPHIE}

AMMAR, A. \& HASSAN, R. M. (2017). « Talking it through: Collaborative Dialogue and Second Language Learning ». Language learning.

BESSON, M.-J. et al. (1979). Mâitrise du français. Lausanne : Office romand des éditions et du matériel scolaires.

BRU, M., ALTET, M. \& BLANCHARD-LAVILLE, C. (2004). « À la recherche des processus caractéristiques des pratiques enseignantes dans leurs rapports aux apprentissages ». Revue française de pédagogie 148, p. 75-89. En ligne : http://ife.ens-lyon.fr/publications/edition-electronique/revuefrancaise-de-pedagogie/INRP_RF148_7.pdf.

BULEA BRONCKART, E. (2015). « À grammaire rénovée, exercices... ? » La Lettre de l'AIRDF 57, p. 39-45. En ligne : https://archive-ouverte.unige.ch/unige:81349.

CHARTRAND, S.-G. \& SIMARD, C. (2000). Grammaire de base. Saint-Laurent : ERPI.

CHARTRAND, S.-G. et al. (1999). Grammaire pédagogique du français d'aujourd'hui. Montréal : Graficor. 
CHERVEL, A. (1977). Et il fallut apprendre à écrire à tous les petits français. Histoire de la grammaire scolaire. Paris : Payot.

COGIS, D. et al. (2016). « L'enseignement de l'orthographe grammaticale ». In : Chartrand, S.-G. (dir.), Mieux enseigner la grammaire. Paris/Montréal : Pearson/Erpi, p. 123-145.

COGIS, D., FISHER, C. \& NADEAU, M. (2015). « Quand la dictée devient un dispositif d'apprentissage ». Glottopol 26, p. 69-91. En ligne : http://glottopol.univ-rouen.fr/telecharger/numero_26/ gpl26_04cogis_fisher_nadeau.pdf.

combetTes, B. (2011). «Phrase et proposition. Histoire et évolution de deux notions grammaticales ». Le français aujourd'hui 2, 173, p. 11-20. En ligne : https://www.cairn.info/revuele-francais-aujourd-hui-2011-2-page-11.htm.

DUBOIS, J. \& LAGANE, R. (1973). La nouvelle grammaire française. Paris : Larousse.

GENEVAY, É. (1994). Ouvrir la grammaire. Lausanne/Montréal : LEP/La Chenelière.

GENEVAY, É. (1996) [1995]. « S'il vous plaît... invente-moi une grammaire !». In : Chartrand, S.-G. (éd.), Pour un nouvel enseignement de la grammaire. Montréal : Éd. Logiques, p. 53-84.

GENOUVRIER, É. \& GRUWEZ, C. (1987). Grammaire pour enseigner le français à l'école élémentaire. Paris : Larousse.

GENOUVRIER, É. \& PEYTARD, J. (1970). Linguistique et enseignement du français. Paris : Larousse.

GIGUÈRE, M.-H. (2015). Effets d'un cercle pédagogique sur le regard professionnel et les pratiques des enseignants $d u 3^{e}$ cycle du primaire en grammaire actuelle. Thèse en sciences de l'éducation : Université de Montréal.

GRÉVISSE, M. (1986, 2016) [1936]. Le Bon usage. Paris : Duculot.

GRÉVISSE, M. (1992) [1953]. Précis de grammaire française. Paris : Duclot.

MINISTÈRE DE L'ÉDUCATION DU QUÉBEC (MEQ) (1995). Programmes d'études. Le français. Enseignement secondaire. Québec : Gouvernement du Québec.

MINISTÈRE DE L'ÉDUCATION DU QUÉBEC (MEQ) (1997a). Errata au programme de français du secondaire. Québec : Gouvernement du Québec.

MINISTÈRE DE L'ÉDUCATION DU QUÉBEC (MEQ) (1997b). Addenda au programme de français du secondaire. Québec : Gouvernement du Québec.

MINISTÈRE DE L'ÉDUCATION ET DE LA SCIENCE (1994). Programmes d'études. Le français. Enseignement primaire. Québec : Gouvernement du Québec.

MINISTÈRE DE L'ÉDUCATION, DES LOISIRS ET DES SPORTS (MELS) (2001). Programme de formation de l'école québécoise. Québec : Gouvernement du Québec. En ligne : http://www.mels.gouv.qc.ca/ $\mathrm{dgfj} / \mathrm{dp} /$ programme_de_formation/primaire/prform2001h.htm.

MINISTÈRE DE L'ÉDUCATION, DES LOISIRS ET DES SPORTS (MELS) (2005). Programme de formation de l'école québécoise : enseignement secondaire, $1^{\text {er }}$ cycle. Chap. 5, Domaine des langues. En ligne : http:// www.mels.gouv.qc.ca/sections/programmeFormation/index.asp?page=secondaire1.

MINISTÈRE DE L'ÉDUCATION, DES LOISIRS ET DES SPORTS (MELS) (2009). Progression des apprentissages en écriture. En ligne : http://www1.mels.gouv.qc.ca/progressionPrimaire/francaisEns/index.asp? page $=$ compEcrire. 
MINISTÈRE DE L'ÉDUCATION, DES LOISIRS ET DES SPORTS (MELS) (2011). Progression des apprentissages au secondaire. Français, langue d'enseignement. En ligne : www.mels.gouv.qc.ca/progression/ secondaire/.

MINISTÈRE DE L'ÉDUCATION, DES LOISIRS ET DES SPORTS (MELS) (2012). Évaluation du plan d'action pour l'amélioration du français, résultats aux épreuves ministérielles d'écriture de juin 2009 et 2010. En ligne : http://www.education.gouv.qc.ca/fileadmin/site_web/documents/dpse/ formation_jeunes/EvalkuationPAAF_2eRapportFinal_ResultatsEpreuves_1.pdf.

NADEAU, M. \& FISHER, C. (2006). La grammaire nouvelle. La comprendre et l'enseigner. Montréal :

G. Morin.

NADEAU, M. \& FISHER, C. (2014). Expérimentation de pratiques innovantes, la dictée 0 faute et la phrase dictée du jour, et étude de leur impact sur la compétence orthographique des élèves en production de texte. Rapport de recherche FQRSC. En ligne : http://www.frqsc.gouv.qc.ca/partenariat/nos-resultatsde-recherche/histoire/experimentation-de-pratiques-innovantes-la-dictee-0-faute-et-la-phrasedictee-du-jour-et-etude-de-leur-impact-sur-la-competence-orthographique-des-eleves-enproduction-de-.

THE NEW TEACHER PROJECT (TNTP), 2015. The Mirage, confronting the hard truth about our quest for teacher development. En ligne : https://tntp.org/assets/documents/TNTP-Mirage_2015.pdf VARGAS, C. (2011). « La maitrise de la langue. Quand les programmes cesseront de tourner en rond ». Le français aujourd'hui 173, p. 113-117.

Wilkinson, K. (2009). « Les effets de la dictée 0 faute sur la compétence en orthographe d'élèves de troisième secondaire ». Mémoire de maîtrise en linguistique, option didactique. Montréal (Québec, Canada) : Université du Québec à Montréal. En ligne : http:// www.archipel.uqam.ca/2597/.

\section{ANNEXES}

\section{Annexe}

Manuels et grammaires du $3^{\mathrm{e}}$ cycle ( $5^{\mathrm{e}}-6^{\mathrm{e}}$ année primaire) consultés.

\section{Avant la progression :}

1. Complices plus (Phare - Mondia - 2003) ; 2. Signet (ERPI - 2003) ; 3. Au-delà des mots (HRW - 2003) ; 4. Cyclades (Modulo - 2003) ; 5. Mordicus (+condensé grammatical) (CEC - 2003) ; 6. Grammaire du 3e cycle (Graficor - 2003) ; 7. Grammaire jeunesse (CEC - 2004) ; 8. Ma première grammaire Bescherelle (Hurtubise - HMH - 2003).

\section{Après la progression :}

11. Les trésors de la grammaire (CEC - 2010); 12. Vingt mille mots sous les mers (CEC - 2010); 13. Clicmots (Grand Duc - 2010) ; 14. Grammaire de base, nouvelle éd. (ERPI - 2011) ; 15. Arobas (ERPI - 2014) ; 16. Charivari (Grand-Duc - 2015) ; 17. Ma grammaire du 3e cycle (L’Envolée - 2015) ; 18. Au programme... en français (Grand Duc - 2010). 


\section{NOTES}

1. Rappelons que ce fut le cas de la Suisse francophone dès les années 1970 (cf. Besson et al., 1979).

2. L'appropriation d'une première langue repose en fait sur des apprentissages implicites, inconscients. Tout enfant de 5 ans au développement normal sait parler, donc produire des phrases, souvent complexes, sans aucune connaissance de la grammaire de sa langue. Ce qui est vrai dans toutes les langues.

3. Pour une présentation de ces deux grammaires, voir M. Nadeau et C. Fisher (2006, chap. 1).

4. Une $12^{\mathrm{e}}$ édition du Bon usage, en 1986, a été remaniée par A. Goosse qui en a modernisé le cadre grammatical, mais cet ouvrage demeure malgré tout le symbole de la grammaire traditionnelle. Une $16^{\mathrm{e}}$ édition est parue en 2016.

5. Cette terminologie reflète la formulation classique de la grammaire générative $: P=S N+S V$ (phrase se réécrit syntagme nominal + syntagme verbal). Nous en discutons plus loin en abordant la question du prédicat.

6. On peut penser à la distinction entre compléments indirects et compléments de phrase qui se présente dans plusieurs cas comme une question de degré dans leur liaison au verbe (cf. Nadeau \& Fisher, 2006, chap. 4).

7. Précisons que trois collaborateurs importants à la conception et rédaction de ce programme étaient des didacticiens spécialistes de la grammaire.

8. Nous nous référons au texte de l'Errata au programme de français au secondaire, paru en janvier 1997 pour remplacer la version originale de la section Grammaire de la phrase et du texte.

9. Elle sera ensuite augmentée pour inclure les autres volets du programme de français. Reconnaissant qu'un tel document répondait à un profond besoin, le Ministère fera établir des progressions pour tous les programmes. Celle pour le français au secondaire paraitra en 2011.

10. La Progression des apprentissages au primaire est organisée en trois séries de tableaux pour chaque aspect (lexique, orthographe d'usage, conjugaison, accords, etc.) : Connaissances, Pistes pour l'appropriation des connaissances, Utilisation des connaissances en écriture. Les premiers "présentent les concepts associés à la description de la langue française et dont la connaissance est nécessaire à l'exercice de la compétence à écrire ». Les seconds " proposent des activités et des pistes de travail pour aider les élèves à construire des concepts, à organiser leurs connaissances, à développer des stratégies». Quant aux derniers, "ils décrivent les comportements susceptibles d'aider les élèves » à se rappeler et à utiliser les connaissances et les stratégies apprises (MELS, 2009, p. 4).

11. Cet examen n'est pas exhaustif faute d'avoir pu consulter toutes les collections, mais il comprend les principales maisons d'édition scolaire québécoises. La liste figure en annexe.

12. Les numéros renvoient à la liste du matériel analysé qui figure en annexe.

13. Au Québec, le matériel didactique de base que les commissions scolaires sont autorisées à acheter doit avoir été approuvé par le Ministère. Les autres ouvrages, dont les cahiers d'exercices périssables, n'ont pas besoin de cette approbation pour être utilisés à titre de matériel « complémentaire » et les éditeurs n'hésitent pas à y annoncer en couverture la conformité avec la Progression des apprentissages, comme s'il y avait eu approbation du Ministère.

14. Entre la phrase de base, la phrase graphique, la phrase syntaxique, la phrase subordonnée, la phrase simple, la phrase complexe, l'élève a de quoi y perdre son latin, d'autant plus que, très souvent, dans les interactions en classe, on ne parle que de " phrase ", sans préciser laquelle.

15. Les cinq critères d'évaluation sont : 1- l'adaptation à la situation de communication ; 2- la cohérence du texte ; 3 - le vocabulaire ; 4- la syntaxe et la ponctuation ; 5- l'orthographe. On les 
retrouve à chaque épreuve d'écriture obligatoire de fin de cycle, mais avec des descriptions et des exigences adaptées au niveau scolaire.

16. Recherche de M. Nadeau, C. Fisher et M.-H. Giguère, financé par le Fonds Québécois de Recherche-Société et Culture (FRQSC) 2016-2019 : Expérimentation de dispositifs didactiques en syntaxe et en ponctuation "à la manière » des dictées métacognitives. Dans sa première phase, ce projet visait à concevoir et à expérimenter des dispositifs innovants en syntaxe-ponctuation. Les effets de ces dispositifs sur la compétence en écriture seront mesurés au cours de la $2^{\mathrm{e}}$ phase du projet, qui débute.

17. C'est la phrase verbale qui est ici travaillée, partant du principe que les grandes régularités sont vues avant les cas plus rares comme la phrase non verbale et que ce sont justement ces régularités qui semblent peu maitrisées par rapport aux attentes des programmes.

\section{RÉSUMÉS}

Cet article fait état de la notion de prédicat au Québec. Depuis une vingtaine d'années, un cadre de grammaire moderne a été adopté par le ministère de l'Éducation dans les programmes de français. En considérant la description de la phrase, cet article retrace d'abord des moments clés de l'introduction de la grammaire nouvelle au primaire et au secondaire dans la succession des programmes officiels. Dans un deuxième temps, la terminologie relative aux constituants de la phrase est examinée dans un échantillon de matériel didactique et de grammaires, faisant ainsi apparaitre l'émergence graduelle de la notion de "prédicat ». Enfin, des exemples tirés d'une recherche en cours illustrent l'utilité des notions de la grammaire moderne, dont celle de prédicat, pour aider les élèves, entre autres, à mieux ponctuer les phrases qu'ils écrivent. La conclusion invite à considérer les conditions d'un changement de pratique en enseignement de la grammaire.

In this paper, we present a general view of the notion of predicat and its implementation in the canadian french speaking province of Quebec. About 20 years ago, a modernised grammar was put forward by the Ministry of Education. Considering the sentence level of description, this paper retraces key moments of the integration of this new grammar in primary and secondary schools' official programs. Subsequently, we examine the evolution of the terminology used to describe the main constituants of the sentence in a sample of didactic material, showing how the term predicat emerged gradually. Finally, we illustrate the utility for the students of this modern description of the sentence which includes the notion of predicat: in an actual research project, an activity was developped to help students in the the punctuation of their texts. We conclude by considering the conditions of professionnal change in the teaching of grammar.

\section{INDEX}

Mots-clés : didactique de la grammaire, grammaire nouvelle, programmes de français, prédicat, utilité de la grammaire

Keywords : didactic of grammar, modern grammar, school curriculum, predicate, utility of grammar 


\section{AUTEURS}

\section{CAROLE FISHER}

Université du Québec à Chicoutimi, CA-G7H 2B1, Canada

\section{MARIE NADEAU}

Université du Québec à Montréal, CA-H3C 3P8, Canada 